\title{
Cognitive modeling as a means of assessment and formation of systemic thinking
}

\author{
Tatiana Ledashcheva*, Vladimir Pinaev \\ Peoples Friendship University of Russia (RUDN University), Faculty of Ecology, 6 Miklukho- \\ Maklaya Street, Moscow, 117198, Russian Federation
}

\begin{abstract}
The article is devoted to the development of systems thinking of students. Systems thinking nowadays becomes crucial for specialists in the field of environmental management. However, the peculiarities of modern education since school are not conducive to the widespread formation of system thinking skills. The authors propose to use cognitive modeling to develop systems thinking in students, as well as to assess the degree of formation of these skills.
\end{abstract}

Systems thinking, in modern time, becomes crucial for specialists in the fields of technic and technology, economics, management, environmental management, etc. In practical activities system analysis started to be applied from the middle of last century. So, president Johnson ordered to implement system analysis in all departments of US federal government, in Austria in 1972 an International institute of applied system analysis (IIASA) was founded for system analysis of global problems [1]. Later imagen of system approach was introduced to solving of specific problems and finally concept of system thinking was formed. According to one of the leading Russian theorist of systemology Pranghishvili I.V. [2] «Aim of system thinking - correctly and coherently accept the environment, integrally conceptualize visions and understand laws and principles of substantial and non-substantial words, learn to implement this laws and concepts, first of all during construction and management of complex systems»»

Herein already in 20th of XX century well known British philosopher A.N. Whitehead declared necessity to train human system thinking as main aim of education system. Vice president of the American Federation of Teachers A.Urbanski named the thinking training - the base of teaching in the future [3]. This can be called the concept of the education system in the XXI century.

All the more necessary is systems thinking for specialists in environmental protection and HSE-management.

Although thinking is a mental process, concept «systems thinking» is not a psychological term and appears only in work on experimental psychology. More widely this concept is considered in scientific works on pedagogy, since the last quarter of the XX century. Last years also grate amount of publications on systems thinking forming at different groups of students. This indicates, from one side, understanding of system thinking necessity, on the flip side - absence of common methodologies for solution of this

* Corresponding author : tledascheva@mail.ru 
problem. Many works, as [4], [5], [6] and others, are dedicated to the concept of systems thinking, validation of its necessity, determination of systems thinking level assessment criterion, but not the methodologies of its formation. However, these studies usually concern technical majors; we were unable to find studies and methodologies suitable for environmental science students.

One of the founders of the introduction of systems thinking into various fields is B. Richmond. He identified and discussed in detail the seven most important skills of systems thinking, which are closely related to each other [7]:

1. dynamic thinking - the ability to see and analyze the behavior of the system in development, rather than concentrating on individual events;

2. closed-loop thinking - the ability to see the cycles of feedbacks responsible for a certain behavior of the system;

3. generic thinking - the ability to see general tendencies, connected with the internal logic of the system development, behind separate events and phenomena generating them;

4. structural thinking - an ability to analyze and correctly model the structure of the system;

5. operational thinking - ability to understand and model the processes occurring in the system on the whole scale;

6. continuum thinking - ability to take into account and predict not only a concrete list of alternatives, but also the whole variety of intermediate possibilities;

7. scientific thinking - the ability to investigate a problem by proposing and approving a hypothesis.

The authors of articles devoted to the formation of systems thinking in recent years provide lists of skills in which systems thinking can be identified [4], [9]. However, regardless of the terminological differences, they are all based on the skills listed above.

We set ourselves the task to develop such a methodology for the formation of system thinking in students that should:

- be universal or little dependent on students' training and specialization;

- provide sustainable formation of systemic thinking, which is connected, first of all, with the future professional activity of students;

-be easily implementable in the existing educational system.

We proposed methodology of system thinking formation, based on methodology of complex semi structured systems formation. Such are social, social-economics, and socioenvironmental-economics systems. Currently, a methodology of modelling and analysis of such systems in the form of weighted oriented graphs is developed, which allows to combine quantitative and qualitative characteristics of object under consideration, to get access to its state, to offer promising ways of development or exit from the crisis state/

Modelling process includes following steps: [10]

1. Problem (aims) formulation.

2. Factors identification (subsystems), covering all aspects of system functioning in the frame of task identified; factors are displayed as vertices of oriented graphs.

3. Each factor receives numerical characteristic (indicator) value of it can be measured in some way.

4. Identification of direct (immediate) links between factors, which are displayed as arcs of oriented graph.

5. Examination of oriented graph strongly connection, including identification of inverse reaction cycles.

6. Assignment of " + " or " - " signs to arcs depending on the nature of factors influence.

7. Weight coefficients assessment of oriented graph arcs by methodologies of straight calculation, statistical or expert estimation. 
8. Calculation of impulse process and system reaction on single disturbances, including check of system stability.

9. Calculation of system factors weights, characterizing impact of each factor on the whole system; analysis of received weights, assessment of system state

10. Management proposal formation, calculation of model response to the proposed management decisions.

Processes of any level can be modelled in such manner - from family to region.

We offer our students to create their own model of any interesting system or process. After the student receives the model in the form of a oriented graph (steps 1-6), the group discusses and clarifies the model. After the student receives the model in the form of a oriented graph (steps 1-6), the group discusses and clarifies the model. After the discussion, the student performs the 7th stage, which requires not only special knowledge on the chosen topic for the calculation of the weight coefficient of system connections, but also allows to improve the skills of information collection, the use of statistical analysis methodology and the organization of interviews. Then, after simple calculations, usually with the help of computer tools (Excel), and analysis of the results, the student again presents his model and conclusions. So proposed course of cognitive modeling realizes project approach, uses visually-vivid thinking and brain storming methodology, implement knowledge received by students during other classes. Herewith consistently critical skills formation is performed: structural thinking, operational thinking, cyclic thinking etc.

Approbation of proposed course in common with improvement of modelling methodology during more than 15 years was performed in different higher educational institutions: Ecological faculty of RUDN University, Russian International Tourism Academy, Russian Presidential Academy of National Economy and Public Administration. During this time, students have developed many models of complex systems to solve a variety of problems.

Since the course is focused on the formation of practical skills of formal modeling and system analysis, the theoretical part of the course contains a minimum of necessary information on system analysis, the theory of oriented graphs and statistical analysis. Therefore, the success of the course depends little on the level of mathematical training of students, which allows it to be used in teaching students of a wide range of areas of study.

In [11] we have analyzed in detail the main mistakes students make when faced with cognitive modeling techniques for the first time. In the course of the subsequent open discussion, these errors are mostly eliminated. It is noteworthy that if the first of the proposed models requires mainly the attention and comments of the teacher, then the discussion of the following models, the initiative goes to the students - experts. This is especially noticeable if the discussion takes place in the form of an Internet forum.

In recent years, however, we have noticed a disturbing trend: models built by students in recent years initially contain fewer vertices than those built by students 10 years ago. There is no need to talk about excessive detail, as it was before, the main attention is now given to a detailed breakdown of each system, after which the number of vertices increases by $1.5-2$ times.

In this case, we are usually not talking about some highly specialized areas in which students simply do not have enough information. This could be, for example, the task of determining the factors affecting academic performance. Such ideas are repeated from year to year, but recently students have found it difficult to name more than 5-6 factors.

Thus, we believe that the task of constructing a cognitive model of any non-specialized problem (or, in a simplified version, the task of selecting factors for such a model) can serve as a good indicator of the formation of systemic thinking skills.

After analyzing the changes in school and higher education in recent years, we concluded that such changes may be related to: 
- The introduction of test examinations and tests, without the need to explain one's position;

- the regulation of the most part of academic disciplines, when students have to memorize rules and particular cases rather than learn to apply the general rule to different tasks, reasoning and generalizing;

-The reduction in the amount of time allocated to part of the disciplines, so there may not be enough time to make interdisciplinary connections;

- introduction of modular system in higher education, when each discipline is read in a very intensive mode but for a short time, which prevents to relate information from different disciplines taught in parallel and, again, to establish interdisciplinary links.

Under these conditions, we consider it extremely necessary to introduce courses that form interdisciplinary connections and systemic thinking, such as the course of cognitive modeling. The course can also be combined with a course on logic and the methodology of scientific creativity. The best place for the proposed course, in our opinion, is the third year of study. This guarantees, at one hand sufficient level of common and specialized disciplines for selection and solving of tasks in the professional sphere, at the other hand there is enough time for implementation and stabilization of system thinking skills in development of dissertation.

\section{References}

1. M.C. Jackson, Problems of Governance, 2(4), 50-67 (2011)

2. I.V. Pranghishvili, Control Sciences, 1 (1), 3-7 (2003)

3. A.Urbanski, Director Shkoly, 4, 79-87 (1994)

4. A.V. Panov, M.A. Fedorova, Omsk Scientific Bulletin, 4 (131),162-165 (2014)

5. J.Raven, Pedagogy and Psychology 3(20), 208-213 (2017)

6. S.D. Makarova, A.A. Otdelkina A.A., T.M. Novokreshchenova, Vestnik of Lobachevsky University of Nizhni Novgorod, 3-4, 123-127 (2014)

7. B. Richmond, Systems dynamics Review, 9(2), 113-133 (1993)

8. B. Richmond, The "thinking" in systems thinking: Seven essential skills. (Pegasus Communications, 2000)

9. I.A. Sychev, Modern problems of science and education. 6, 286 (2013)

10. V. Pinaev, T. Ledashcheva: E3S Web of Conferences, 169, 02020 (2020)

11. T. Ledashcheva, V.E. PinaevV.I. GorelovO.L. Karelova, Proceedings of the International Science and Technology Conference "FarEastCon" (ISCFEC 2019) (Atlantis press, 2019) 